\title{
Tests de germination et de croissance de Artemisia annua L. anamed sur différents substrats au Bénin
}

\author{
Mariatou SOUNON, Romain GLELE KAKAÏ ${ }^{*}$, Julien AVAKOUDJO, \\ Achille E. ASSOGBADJO et Brice SINSIN \\ Laboratoire d'écologie appliquée, Faculté des Sciences Agronomiques, \\ Université d'Abomey-Calavi, 01 BP 526 Cotonou, Bénin. \\ *Corresponding author, Email: gleleromain@yahoo.fr, Tel.: 0022995840800
}

\section{RESUME}

La présente étude a porté sur la germination et la croissance de la variété améliorée anamed de l'armoise annuelle, Artemisia аппиа L. une espèce antipaludique d'origine chinoise. L'objectif était d'identifier la meilleure technique de production des plants de l'espèce au Bénin et en Afrique. A cet effet des tests de germination des graines de A. аппиа $\mathrm{L}$. anamed ont été effectués sur une durée de deux semaines dans des germoirs traditionnels et modernes. Par ailleurs, les plants issus des germoirs ont été repiqués dans des pots de substrat sableux et ferrallitique afin d'identifier le meilleur substrat pour une bonne croissance des plants en pépinière. De plus, ces plants ont été transplantés sur terre ferme à trois différents écartements afin de déterminer la densité appropriée pour la plantation de l'espèce. Il ressort des résultats obtenus que les germoirs traditionnels se révèlent meilleurs pour le test de germination des graines de l'espèce (taux de germination de $84,5 \%)$. Aussi, le sol ferralitique s'est montré globalement plus approprié pour la production en pépinière de l'espèce $(9,1 \mathrm{~cm}, 2,6 \mathrm{~mm}, 4,9 \mathrm{~cm}$ et 14 respectivement pour la hauteur moyenne des plants, le diamètre moyen au collet, la largeur moyenne des feuilles et le nombre moyen de feuilles par plant à 28 jours). Le sol ferralitique se révèle aussi efficace pour la vitesse de croissance des plants surtout en ce qui concerne le nombre de feuilles et leur largeur. Le rendement en biomasse foliaire de l'espèce est plus élevé en plantation pour un écartement de $0,8 \mathrm{~m} \mathrm{x} 0,6 \mathrm{~m}\left(312,4 \mathrm{~g} / \mathrm{m}^{2}\right)$. Une bonne production de l'espèce est alors possible sur sol ferralitique avec des écartements de $0,8 \mathrm{~m}$ x $0,6 \mathrm{~m}$.

() 2009 International Formulae Group. All rights reserved.

Mots-clés: Artemisia апnиа L. anamed, technique de reproduction, germoir, substrat, plantation.

\section{INTRODUCTION}

Le paludisme est une maladie parasitaire des régions tropicales due au Plasmodium falciparum transmis par les moustiques de type anophèle (Lacroix, 2004). Chaque année, environ 500 millions de personnes contractent cette maladie dans le monde entier dont trois millions en meurent (Hans, 2001). L'Organisation Mondiale de la Santé (2006) estime que $80 \%$ des cas de décès dus au paludisme sont enregistrés en Afrique subsaharienne. Les traitements utilisés sont nombreux et deviennent caduques car la résistance de $P$. falciparum aux antimalariques synthétiques, liée à la résistance des moustiques vecteurs aux insecticides, a ouvert la voie à la recherche de nouveaux agents antipaludiques. Le nouveau traitement qui donne des résultats très satisfaisants est à base de Artemisia апnиа (Ferreira et Janick, 1995).

Artemisia аппиа est une espèce continentale d'Asie de la famille des Asteraceae et trouvée dans les zones tempérées, sur sol sableux des bords de fleuves (Meusel et Jäger, 1992). Elle peut atteindre plus de $3 \mathrm{~m}$ de haut en culture (Hans, 2003). C'est un arbuste aromatique dont le goût est fortement amer pouvant créer un avortement chez les femmes enceintes (Hans 
et Keith, 2006). Les feuilles sont biparipennées et dentelées de couleur vert foncée ou vert-claire. La tige très ramifiée a une longueur comprise entre 30 et $100 \mathrm{~cm}$. La floraison est estivale. Le cycle de développement de cette espèce dure environ un an (Guyonnet, 2005). A Madagascar et au Cameroun, la plante séjourne sept semaines en pré-pépinière et pépinière, et cinq mois en plantation (Dreuilhe, 2005). La multiplication de $A$. аппиа anamed se fait par graine (semis au germoir suivi de repiquage) ou par bouture (taux de réussite de 95 à 100\%). Les graines sont viables pendant au moins 3 ans en conditions sèches (Ferreira et al., 1997).

Depuis près de 2000 ans, elle est utilisée en Chine comme antipaludéen (Klayman, 1993). Ses feuilles contiennent en effet une lactone sesquiterpénique endoperscide (artemisinine) et une autre substance active (dihydroartemisinine) très efficace contre les souches parasites responsables du paludisme. Elle revêt ainsi une grande importance dans la recherche pharmaceutique contre le paludisme (Ferreira et Janick, 1995). Une variété améliorée de l'espèce (A. апnиa anamed) est mise au point par le Centre International de Recherche en Agroforesterie (ICRAF) à partir des graines fournies par l'action médecine naturelle (anamed) et qui s'adapte à de nombreux types de sols. La bonne maîtrise de la production de A. annua à tout moment de l'année en Afrique Sub-Saharienne (zone de forte prévalence du paludisme) permettra de lutter efficacement contre la maladie.

L'objectif principal de cette étude était alors d'évaluer la dynamique de germination, de croissance et de production de $A$. аппиа anamed sur deux différents types de substrats du Sud-Bénin suivant différents écartements pour une bonne production de la plante. La première hypothèse testée dans l'étude était relative à un effet non significatif du type de germoir (moderne et traditionnel) sur le taux de germination de $A$. аnnua; la deuxième hypothèse est liée à un effet non significatif du substrat (ferralitique et sableux) sur les paramètres de croissance de l'espèce en pépinière ; la troisième hypothèse est relative à l'effet non significatif de l'écartement $(1 \mathrm{~m}$ x $1 \mathrm{~m} ; 0,8 \mathrm{~m} \mathrm{x} 0,8 \mathrm{~m}$ et $0,8 \mathrm{~m} \mathrm{x} 0,6 \mathrm{~m})$ des plants sur les paramètres de croissance et de productivité des plants de l'espèce.

\section{MATERIEL ET METHODES Matériel}

Le matériel utilisé est composé principalement du matériel végétal et des substrats. Le matériel végétal utilisé est constitué de deux emballages d'un gramme constitués chacun de 5000 semences de $A$. annua anamed mélangées avec de la poudre, le tout mis dans une boite. Ces graines sont particulièrement très petites et leur poids moyen est de $0,07 \mathrm{mg} /$ graine. Deux substrats sont considérés à savoir le sol sableux de Sèmè-Kpodji (au Sud-Est du Bénin) et le sol ferralitique d'Abomey-Calavi (au Sud-Est du Bénin) dont les caractéristiques sont données au tableau 1. L'analyse de ce tableau révèle que le substrat ferralitique est moins acide avec des valeurs plus élevées pour la plupart des caractéristiques physico-chimiques par rapport au substrat sableux, notamment des concentrations en cations échangeables et la somme des bases plus élevées. Pour le test de germination, les deux substrats sont préalablement débarrassés de toutes impuretés visibles à l'œil nu. Ils sont ensuite stérilisés pour détruire les bactéries, les champignons et les graines de mauvaises herbes qui pourraient s'y trouver. D'autres matériels sont utilisés à savoir le matériel de mesure (pied à coulisse, double-décimètre), le sable de mer utilisé à la phase de germination, les pots en plastiques utilisés pour le test de pépinière, les sachets de polyéthylène pour le repiquage des plantules, le pulvérisateur et l'arrosoir.

\section{Description du site d'expérimentation}

Les essais de germination et de plantation sont conduits à la Ferme d'Application et de Production (FAP) de la Faculté des Sciences Agronomiques (FSA) de l'Université d'Abomey-Calavi (UAC). Le site est situé au nord de la ville de Cotonou dans la commune d'Abomey-Calavi. Le climat de la localité est de type guinéen caractérisé par deux saisons pluvieuses et deux saisons sèches. La grande saison pluvieuse couvre la période de mi-mars à fin juillet et la petite saison de mi-septembre à mi-novembre. La grande saison sèche s'étale sur la période allant de mi-novembre à mi-mars et la petite du mois d'août à mi-septembre. La pluviométrie moyenne annuelle est de 1200 $\mathrm{mm}$, la température moyenne annuelle est de $28{ }^{\circ} \mathrm{C}$ tandis que l'humidité relative moyenne 
Tableau 1: Caractéristiques physico-chimiques des substrats.

\begin{tabular}{lcc}
\hline Caractéristiques & $\begin{array}{c}\text { Substrat ferralitique } \\
\text { d'Abomey-Calavi }\end{array}$ & $\begin{array}{c}\text { Substrat sableux } \\
\text { de Sèmè-Kpodji }\end{array}$ \\
\hline pHeau & 5,85 & 6,70 \\
pHkcl & 5,10 & 4,90 \\
$\mathrm{~N}$ total $(\%)$ & 0,11 & 0,04 \\
$\mathrm{C}$ total $(\%)$ & 0,96 & 0,46 \\
$\mathrm{MO}(\%)$ & 1,66 & 0,79 \\
$\mathrm{C} / \mathrm{N}$ & 9,11 & 12,00 \\
$\mathrm{P}(\mathrm{ppm})$ & 2,54 & - \\
$\mathrm{CEC}($ méq / 100g) & 9,00 & 3,30 \\
$\mathrm{Ca}++$ (méq / 100g) & 3,07 & 1,35 \\
Mg++ (méq /100g) & 1,06 & 0,45 \\
$\mathrm{~K}+($ méq / 100g) & 0,82 & 0,26 \\
Na+ (méq / 100g) & 0,74 & 0,33 \\
Somme des bases & 5,69 & 2,39 \\
\hline $\mathrm{N}$ total: azote total; C total : carbone total; MO: matière organique; C/N: Rapport carbone/azote; P: phosphore; \\
CE : cation échangeable; Ca++: carbone; Mg++ : magnésium (cation); K+: potassium (cation); Na : sodium \\
(cation) ; Sources: Sogansa (2003).
\end{tabular}

est de $80 \%$. Les travaux d'expérimentation sont menés sur ce site de décembre 2006 à mimai 2007.

\section{Méthodes}

Test de germination de A. annua anamed

La poudre de semences de A. аппиа anamed est mélangée à du sable de mer de façon à obtenir $60 \mathrm{~g}(1 \mathrm{~g}$ de poudre de semences pour $59 \mathrm{~g}$ de sable). Deux lots de mélange sont constitués. Une quantité de $50 \mathrm{~g}$ de ce mélange est considérée pour les germoirs traditionnels et $6 \mathrm{~g}$ du second lot de $10 \mathrm{~g}$ est utilisé pour les germoirs artificiels ou jiffy-pots. Les germoirs traditionnels sont longs de $50 \mathrm{~cm}$ et larges de $35 \mathrm{~cm}$ confectionnés à l'aide des planches en bois au niveau local. Ils ont une surface de base approximative de $1750 \mathrm{~cm}^{2}$ et une hauteur de $25 \mathrm{~cm}$ tandis que les germoirs artificiels sont sous forme de comprimés de couleur brune, fabriqués en fibres de noix de coco, de forme circulaire de $3,7 \mathrm{~cm}$ de diamètre, soit une surface de base de $10,74 \mathrm{~cm}^{2}$ avec déjà un substrat pour le semis des graines. Le lot de $50 \mathrm{~g}$ est divisé en 5 sous-lots qui contiennent chacun 833 graines; 4 sous-lots sont utilisés pour parsemer 4 germoirs traditionnels soit une densité de 0,48 graine $/ \mathrm{cm}^{2}$. Les 4 souslots du $2^{\text {ème }}$ lot sont utilisés pour parsemer les 4 germoirs modernes à raison de 12 graines par germoir, soit une densité de 1,12 graine $/ \mathrm{cm}^{2}$ selon les recommandations du fabricant. Dans deux germoirs traditionnels est mis le substrat ferralitique d'AbomeyCalavi et dans les deux autres est mis le substrat sableux de Sèmè Kpodji. Le dispositif expérimental utilisé est complètement aléatoire et les mesures sont effectuées trois jours après le semis des graines; le nombre de graines de A. аппиа anamed ayant germé est compté quotidiennement avec une loupe et ceci jusqu'au stade de quatre feuilles, à deux semaines après semis.

Le pourcentage du nombre cumulatif de graines germées est déterminé chaque jour. Des nuages de points sont établis et expriment le pourcentage de germination en fonction du nombre de jours après semis. Pour la plupart des unités expérimentales, l'allure observée est du type exponentiel de la forme :

$T=100-a \exp (-b \mathrm{Nj})$,

$T=$ taux de germination et $\mathrm{Nj}=$ nombre de jours après semis.

Ce modèle est ajusté aux données de taux de germination et de nombre de jours après semis de chaque unité expérimentale pour déterminer les paramètres $a$ et $b$ de l'équation (1). Dans cette équation le paramètre $a$ est lié au taux de germination initiale (au $3^{\text {ème }}$ jour après semis) et $b$ donne une idée de la vitesse exponentielle de germination. Une analyse de la variance à un 
critère de classification est ensuite effectuée sur les paramètres $a$ et $b$ pour comparer les deux substrats en utilisant le logiciel SAS (SAS, 1999).

Test de caractérisation agronomique en pépinière

Après l'étape de germination, 120 plants sont transplantés dans les pots en plastique afin d'identifier le substrat le plus approprié à la pépinière pour $A$. апnиa. Les pots sont disposés en blocs aléatoires complets (BAC) comme le montre la figure 1 . Chaque unité expérimentale comporte 15 plants. $\mathrm{Au}$ niveau de chaque unité expérimentale (pot), les variables de croissance des plantes sont mesurées chaque semaine pendant 4 semaines. Il s'agit de la hauteur totale, du diamètre au collet, du nombre de feuilles, de la longueur des feuilles et de la distance des entre-nœuds de chaque plant.

Les moyennes et coefficients de variation de chaque paramètre de croissance sont calculés de la $1^{\text {ère }}$ à la $4^{\text {ème }}$ semaine et pour chaque type de substrat. De plus, des nuages de points représentant la variation de chaque paramètre en fonction du nombre de jours après mise en pépinière sont établis et ont permis de noter que dans la plupart des cas, la tendance d'évolution des paramètres est linéaire :

$$
y=a+b \mathrm{Nj}
$$

Les coefficients de régression $a$ et $b$ ont été estimés pour chaque plant et chaque variable de croissance par une régression linéaire simple. Une analyse de la variance est ensuite effectuée en utilisant le modèle mixte pour comparer les deux substrats suivant les valeurs des paramètres $a$ et $b$ en utilisant le logiciel SAS. Dans ce modèle, les blocs constituent le facteur aléatoire alors que le facteur substrat est fixe. Par ailleurs, le paramètre $a$ est lié à la valeur initiale des paramètres de croissance des plants alors que $b$ représente leur vitesse de croissance.

\section{Caractéristique agronomique en plantation}

Après un séjour d'un mois à la pépinière, les plants sont mis en terre à la ferme de la FSA. Trois lots de 60 plants sont constitués; les plants du premier lot sont transplantés à intervalle de $1 \mathrm{~m}$ x $1 \mathrm{~m}(4$ plants $/ \mathrm{m}^{2}$ ), le second lot à intervalle de $0,8 \mathrm{~m}$ x $0,8 \mathrm{~m}\left(6,25\right.$ plants $\left./ \mathrm{m}^{2}\right)$ et les 60 plants restants à intervalle de $0,8 \mathrm{~m}$ x $0,6 \mathrm{~m}(8,33$ plants $/ \mathrm{m}^{2}$ ). Chaque lot de 60 plants est subdivisé en 2 sous-lots de 30 plants issus de chacun des deux substrats. Le dispositif expérimental utilisé est en blocs aléatoires complets. Ce dispositif est destiné à évaluer l'effet de l'écartement sur la croissance des plants de $A$. annua anamed.

Toutes les semaines, les paramètres biologiques suivants ont été mesurés sur les 180 plants pour un suivi rigoureux à la plantation: le diamètre au collet, la hauteur totale, la distance des entre-nœuds. Les nuages de points établis pour la hauteur et la distance entre-nœuds en fonction du nombre de jours indiquent globalement une tendance exponentielle dont l'équation est :

$$
y=a \exp (b \mathrm{Nj})
$$

Dans l'expression (3), $y$ est le paramètre de croissance mesuré, $a$ représente les conditions initiales de croissance et $b$ est lié à la vitesse exponentielle de croissance des paramètres. En ce qui concerne le diamètre au collet (Dc), on a noté une tendance logarithmique dont l'équation est :

$$
y=a^{\prime}+b^{\prime} \ln (\mathrm{Nj})
$$

Les valeurs de ces deux paramètres sont ensuite soumises à une analyse de variance à trois facteurs (blocs, écartement, substrat) en utilisant le modèle mixte. Le test de Student Newman et Keuls a été effectué pour structurer les moyennes. Le logiciel SAS (SAS, 1999) a été utilisé pour le traitement des données.

\section{Récolte et séchage des feuilles de A. annua anamed}

Après l'expérimentation, les plants entiers sont déterrés; la partie racinaire est coupée avec le sécateur; les feuilles sont récoltées et sont mises dans des enveloppes, puis encodées et pesées. Après séchage à l'étuve à $40{ }^{\circ} \mathrm{C}$ durant trois jours, les feuilles sont transformées en thé. Le thé obtenu est mis dans des sachets qui sont déposés à l'abri de la lumière et de l'humidité (30 à $40{ }^{\circ} \mathrm{C}$ ). Par la suite, le poids sec des feuilles, tige et racines des plants de $A$. annua est calculé.

\section{RESULTATS}

Dynamique de germination de $A$. annua anamed

Le modèle traduisant l'évolution du taux de germination $(T)$ des graines de $A$. annua anamed en fonction du nombre de jours 

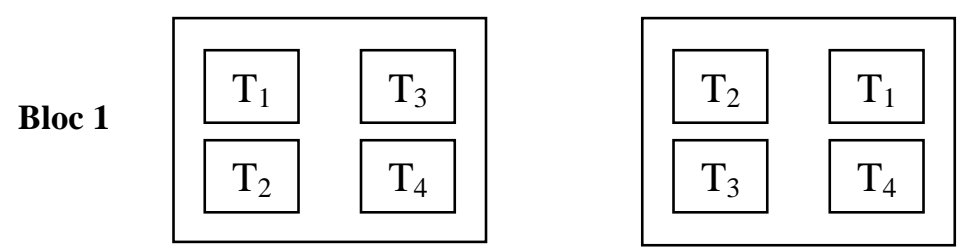

Bloc 2

Figure 1: Dispositif expérimental en Blocs aléatoires complets utilisés pour le test de pépinière dans les pots et sachets. Légende : $T_{1}$ : plants du germoir artificiel transplantés sur le substrat sableux; $T_{2}:$ plants du germoir traditionnel transplantés sur le substrat sableux; $\mathrm{T}_{3}$ : plants du germoir artificiel transplantés sur le substrat ferralitique; $\mathrm{T}_{4}$ : plants du germoir traditionnel transplantés sur le substrat ferralitique.

$(\mathrm{Nj})$ après semis est traduit par l'équation (1). Le tableau 2 présente les taux moyens de germination selon le type de germoir

Il ressort de l'analyse du tableau 2 que le taux moyen de germination est plus important $(84,5 \%)$ pour les germoirs traditionnels en bois alors qu'il est faible $(40,4 \%)$ pour les germoirs modernes. L'effet $\mathrm{du}$ substrat sur la dynamique de germination de l'espèce est décrit à travers la moyenne et le coefficient de variation du paramètre $a$ (fraction maximale théorique de germination) et du paramètre $b$, lié à la vitesse exponentielle de germination (tableau 3). La fraction maximale théorique de germination est plus importante pour les germoirs traditionnels en bois que pour les germoirs modernes (tableau 3). En ce qui concerne le paramètre $b$, elle est plus élevée pour les germoirs traditionnels en bois que pour les germoirs artificiels modernes.

Ces résultats montrent que la dynamique de germination des graines de $A$. annua L. anamed n'est pas la même d'un germoir à l'autre. Cette dynamique de germination est illustrée par la figure 2. Son analyse permet de constater que le taux de germination est plus important pour les germoirs modernes que pour les germoirs traditionnels en bois durant les deux premiers jours. Du $3^{\text {ème }}$ au $14^{\text {ème }}$ jour, le taux et la vitesse de germination sont devenus plus importants au niveau des germoirs traditionnels en bois.

\section{Caractéristiques agronomiques}

Effet du substrat sur la croissance des plants en pépinière

Les moyennes et coefficients de variation des caractéristiques agronomiques des plants en pépinière selon le type de germoir et de substrat sont présentés au tableau 4. Son analyse révèle que la hauteur des plants est plus élevée dans les germoirs traditionnels que dans les germoirs modernes et sur sol ferrallitique que sur sol sableux.

Le diamètre au collet, le nombre et la longueur des feuilles des plants ne varient pas significativement d'un germoir à un autre. Toutefois, les valeurs les plus élevées de ces paramètres sont notées sur sol ferrallitique. En effet, on déduit du tableau 4 que la hauteur moyenne des plants, le diamètre moyen au collet, la longueur moyenne des feuilles et le nombre moyen de feuilles par plant à 28 jours sont respectivement de $9,1 \mathrm{~cm}, 2,6 \mathrm{~mm}, 4,9$ $\mathrm{cm}$ et 14 sur sol ferralitique contre $7,1 \mathrm{~cm}$, $1,9 \mathrm{~mm}, 3,7 \mathrm{~cm}$ et 10 respectivement pour les mêmes paramètres sur sol sableux.

L'effet du substrat sur la croissance des plants selon ces quatre paramètres est résumé au tableau 5 qui présente la moyenne et le coefficient de variation des deux paramètres $a$ (valeur initiale du paramètre) et $b$ (vitesse de croissance) de l'équation (2).

Il ressort de l'analyse du tableau 5 qu'en ce qui concerne la hauteur des plants, on ne note pas de différence significative entre sol sableux et sol ferrallitique suivant la hauteur initiale et la vitesse de croissance en hauteur. Par contre, dans le cas du diamètre au collet, on peut noter que sa valeur initiale (paramètre $a$ ) est significativement plus importante sur sol ferrallitique que sur sol sableux. Toutefois, la vitesse de croissance est différente pour les deux types de sol. En ce qui concerne les paramètres relatifs aux feuilles de A. аппиа, notamment le nombre de feuilles et la longueur des feuilles, on note une valeur initiale égale pour les deux types de sol. Toutefois, la vitesse de croissance (paramètre $b$ ) est plus élevée sur sol ferrallitique que sur sol sableux. 
Tableau 2: Taux moyens de germination selon le type de germoir (en \%).

\begin{tabular}{|c|c|c|c|c|c|c|c|c|}
\hline \multirow{3}{*}{$\begin{array}{l}\text { Type de germoir } \\
\text { Numéro de germoir }\end{array}$} & \multicolumn{4}{|c|}{ Traditionnels } & \multicolumn{4}{|c|}{ Modernes } \\
\hline & \multicolumn{2}{|c|}{ Sol ferralitique } & \multicolumn{2}{|c|}{ Sol sableux } & \multirow[b]{2}{*}{1} & \multirow{2}{*}{2} & \multirow{2}{*}{3} & \multirow{2}{*}{4} \\
\hline & 1 & 2 & 3 & 4 & & & & \\
\hline Moyenne (\%) & 83,5 & 84,9 & 86,9 & 82,7 & 27 & 48,4 & 36,9 & 49,4 \\
\hline Grande moyenne (\%) & \multicolumn{4}{|c|}{84,5} & \multicolumn{4}{|c|}{40,4} \\
\hline
\end{tabular}

Tableau 3: Moyenne $(m)$ et coefficient de variation $(c v)$ des paramètres d'évolution du pourcentage de germination.

\begin{tabular}{lcccc}
\hline \multirow{2}{*}{ Germoir } & \multicolumn{2}{c}{$\boldsymbol{a}$} & \multicolumn{2}{c}{$\boldsymbol{b}$} \\
\cline { 2 - 5 } & $\boldsymbol{m}(\boldsymbol{\%})$ & $\boldsymbol{c v}(\boldsymbol{\%})$ & $\boldsymbol{m}(\boldsymbol{\%})$ & $\boldsymbol{c v}(\boldsymbol{\%})$ \\
\hline Traditionnel & 318,9 & 68,5 & 0,48 & 36,9 \\
Artificiel & 78,0 & 14,1 & 0,03 & 30,5 \\
Probabilité & \multicolumn{2}{c}{$<0,001$} & \multicolumn{2}{c}{0,001} \\
\hline
\end{tabular}

Tableau 4: Moyenne $(m)$ et coefficient de variation $(c v)$ de caractéristiques des plants en pépinières selon le type de germoir et de substrat.

\begin{tabular}{|c|c|c|c|c|c|c|c|c|c|c|}
\hline \multirow[b]{2}{*}{ Germoir } & \multirow[b]{2}{*}{ Subst. } & \multirow[b]{2}{*}{$\mathbf{N b}$} & \multicolumn{2}{|c|}{ Hauteur (cm) } & \multicolumn{2}{|c|}{ Dc (mm) } & \multicolumn{2}{|c|}{ Nfeuil } & \multicolumn{2}{|c|}{ Lgfeuil (cm) } \\
\hline & & & $m$ & $c v$ & $m$ & $c v$ & $m$ & $c v$ & $m$ & $c v$ \\
\hline 1 & 1 & 7 & 0,9 & 31,3 & - & - & 5,0 & 21,2 & 0,4 & 31,7 \\
\hline 1 & 1 & 14 & 2,5 & 32,3 & 0,9 & 48,4 & 6,9 & 19,5 & 1,3 & 31,2 \\
\hline 1 & 1 & 21 & 3,0 & 33,6 & 1,3 & 34,6 & 7,7 & 19,9 & 2,4 & 27,8 \\
\hline 1 & 1 & 28 & 7,1 & 26,5 & 2,0 & 24,9 & 10,5 & 24,4 & 3,8 & 27,0 \\
\hline 1 & 2 & 7 & 1,6 & 32,5 & - & - & 6,3 & 20,9 & 0,6 & 50,7 \\
\hline 1 & 2 & 14 & 4,0 & 24,0 & 1,3 & 29,7 & 7,1 & 15,3 & 2,0 & 27,3 \\
\hline 1 & 2 & 21 & 4,7 & 22,3 & 1,6 & 27,4 & 7,5 & 15,5 & 3,5 & 21,9 \\
\hline 1 & 2 & 28 & 9,4 & 17,1 & 2,3 & 19,0 & 12,8 & 25,2 & 4,7 & 17,5 \\
\hline 2 & 1 & 7 & 1,2 & 30,4 & - & - & 5,7 & 18,9 & 0,4 & 27,3 \\
\hline 2 & 1 & 14 & 2,5 & 40,2 & 0,8 & 30,7 & 6,7 & 19,0 & 1,4 & 28,2 \\
\hline 2 & 1 & 21 & 2,9 & 41,1 & 1,2 & 34,0 & 7,5 & 23,3 & 2,4 & 39,4 \\
\hline 2 & 1 & 28 & 7,0 & 37,2 & 1,9 & 33,9 & 10,0 & 30,9 & 3,6 & 35,1 \\
\hline 2 & 2 & 7 & 1,6 & 31,0 & - & - & 6,5 & 17,6 & 0,5 & 31,9 \\
\hline 2 & 2 & 14 & 4,2 & 27,2 & 1,5 & 39,8 & 7,7 & 19,3 & 2,0 & 25,8 \\
\hline 2 & 2 & 21 & 4,4 & 22,8 & 1,8 & 27,4 & 9,1 & 20,7 & 3,6 & 26,6 \\
\hline 2 & 2 & 28 & 10,4 & 19,3 & 2,8 & 27,7 & 14,7 & 35,5 & 5,1 & 20,8 \\
\hline
\end{tabular}


Tableau 5: Moyenne $(m)$ et coefficient de variation $(c v)$ du paramètre $a$ ' lié aux conditions initiales de croissance et de la vitesse de croissance $\left(b^{\prime}\right)$ des pépinières de A. апnиa selon le type de substrat.

\begin{tabular}{|c|c|c|c|c|c|c|}
\hline \multirow{2}{*}{ Variable } & \multirow{2}{*}{ Moyenne } & \multirow[b]{2}{*}{ Paramètre } & \multicolumn{2}{|c|}{ Sol sableux } & \multicolumn{2}{|c|}{ Sol ferrallitique } \\
\hline & & & $m$ & $c v$ & $m$ & $c v$ \\
\hline \multirow{2}{*}{ Hauteur $(\mathrm{cm})$} & \multirow{2}{*}{4,3} & $a$ & $-1,2 \mathrm{a}$ & 66,5 & $-1,3 a$ & 64,9 \\
\hline & & $b$ & $0,3 \mathrm{~b}$ & 36,7 & $0,4 \mathrm{a}$ & 21,1 \\
\hline \multirow{2}{*}{$\begin{array}{l}\text { Diamètre au } \\
\text { collet (mm) }\end{array}$} & \multirow{2}{*}{1,6} & $a^{\prime}$ & $-0,3 a$ & 200,8 & $0,1 b$ & 590,8 \\
\hline & & $b^{\prime}$ & $0,1 \mathrm{a}$ & 42,1 & $0,1 \mathrm{a}$ & 38,5 \\
\hline \multirow{2}{*}{ Nfeuil } & \multirow{2}{*}{8,5} & $a$ & $3,7 \mathrm{a}$ & 42,9 & $3,2 \mathrm{a}$ & 69,3 \\
\hline & & $b$ & $0,2 b$ & 59,1 & $0,3 \mathrm{a}$ & 61,7 \\
\hline \multirow{2}{*}{ Lgfeuil (cm) } & \multirow{2}{*}{2,4} & $a$ & $-0,8 \mathrm{a}$ & 57,3 & $-0,9 a$ & 57,5 \\
\hline & & $b$ & $0,1 b$ & 34,6 & $0,2 \mathrm{a}$ & 21,7 \\
\hline
\end{tabular}

Pour chaque paramètre, les chiffres de même lettre ne sont pas significativement différents. Nfeuil: nombre de feuilles ; Lgfeuil: longueur des feuilles.

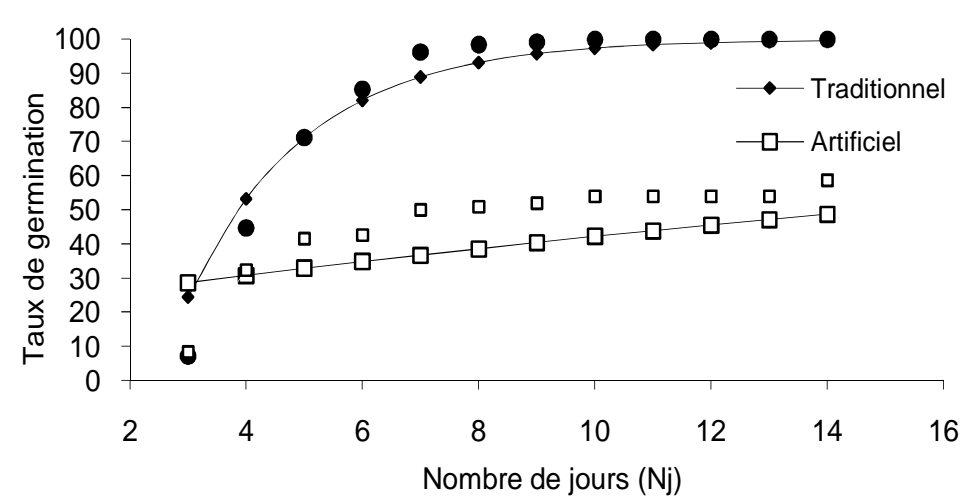

Figure 2: Evolution du taux de germination suivant le nombre de jours après semis.

Effet de l'écartement sur la croissance des plants en plantation

L'effet de l'écartement est évalué en plantation sur terre ferme par rapport à la croissance en hauteur, la croissance de la distance entre les entre-nœuds ainsi que la croissance en diamètre au collet. La tendance évolutive des paramètres est présentée à la figure 3 . On note de cette figure la même tendance de croissance de la hauteur et de la distance entre-nœuds des plants quel que soit l'écartement considéré ( $\mathrm{P}>0,05)$. Par contre, dans le cas du diamètre au collet, la vitesse logarithmique de croissance (paramètre $b$ ') est plus importante pour l'écartement $0,8 \mathrm{~m}$ x 0,6 $\mathrm{m} \quad(\mathrm{P}<0,05)$. Toutefois, la valeur initiale (paramètre $a^{\prime}$ ) est significativement égale pour les trois types d'écartement $(\mathrm{P}>0,05)$. Par ailleurs, la hauteur moyenne, le diamètre moyen au collet et la distance moyenne entrenœuds des plants pour l'ensemble des écartements sont respectivement égaux à 29,5 $\mathrm{cm}, 5,9 \mathrm{~mm}$ et $1 \mathrm{~cm}$

\section{Paramètres de production de $A$. annua anamed}

La matière fraîche et sèche des feuilles, tiges et racines ainsi que la biomasse totale ont été quantifiées suivant le type d'écartement et la moyenne ainsi que le coefficient de variation de ces variables sont présentés au tableau 6.

Les plus fortes productions en feuilles, racines et tiges sont significativement notées pour l'écartement 0,8 m x 0,6 m (tableau 7). Les deux autres écartements donnent pratiquement les mêmes productions en biomasse. 


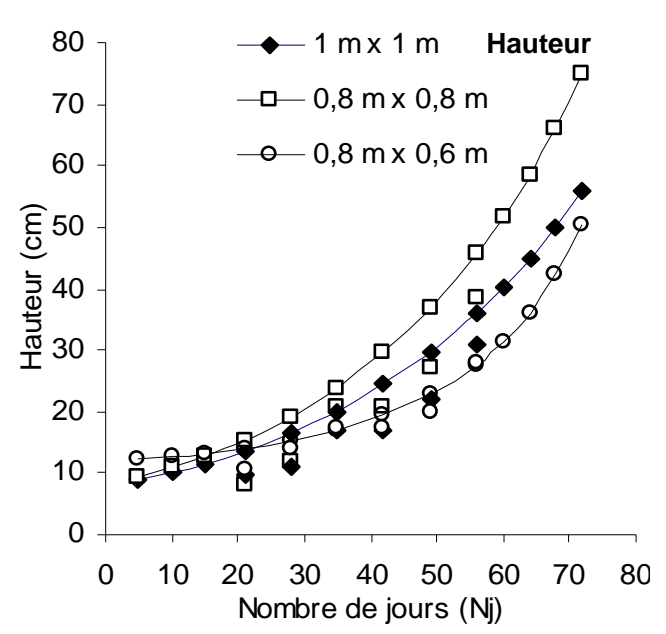

Diamètre au collet

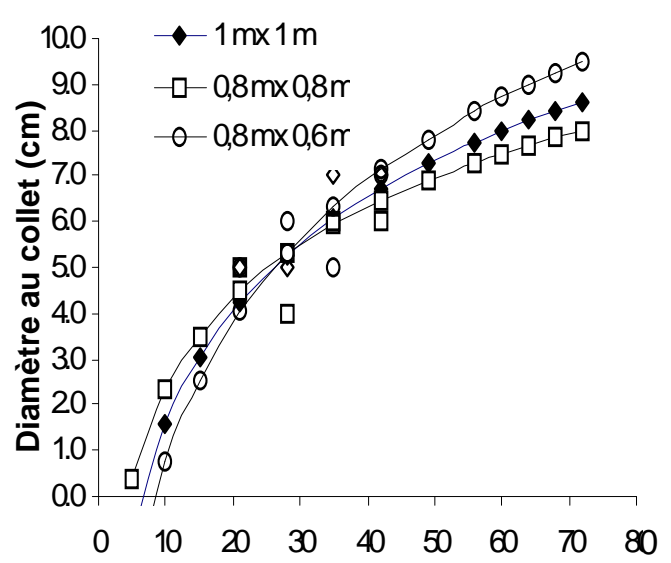

Nombre de jours

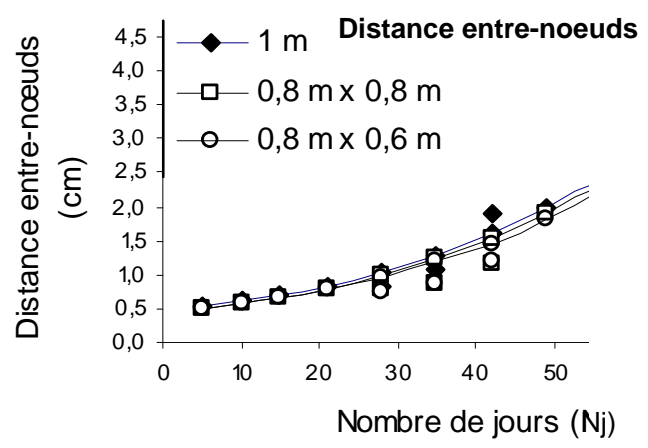

Figure 3: Evolution des paramètres de croissance des plants en fonction du nombre de jours après semis et suivant le type d'écartement à la plantation.

\section{DISCUSSION}

Le test de germination des graines de $A$. аппиа L. anamed dans deux différents types de germoir a montré que le temps maximum de levée des graines dans les conditions de l'étude était de trois jours après semis avec un taux moyen de germination égal à 84,5\% dans les germoirs traditionnels. Le résultat obtenu pour le temps de levée des graines de $A$. апnиа L. anamed est différent de celui obtenu par Barrié (2006) au Campuac en France avec un temps de levée minimum d'un mois. La différence notée s'explique en partie par les conditions climatiques de culture en France, mais aussi le fait que $A$. аппиа L. anamed est une variété améliorée. En ce qui concerne le taux de germination, il est presque total dans les germoirs traditionnels en bois et moyen dans les germoirs modernes (jiffy-pots). Ceci signifie que plus de la moitié des graines restent dans leur état de dormance au niveau des germoirs modernes (jiffy-pots). Ces résultats révèlent l'impact de la densité de semis des graines de A. annua sur la levée de leur dormance. En effet une forte densité de semis réduit l'espace disponible pour la germination de chaque graine et induit alors des effets de compétition entre graines qui peuvent ralentir leur germination comme c'est noté dans les germoirs modernes. La densité de semis des graines recommandée par le fabricant pour les germoirs modernes serait alors élevée pour une bonne levée des graines de $A$. апииа anamed $\left(1,12\right.$ graines $/ \mathrm{cm}^{2}$ contre 0,48 graines $/ \mathrm{cm}^{2}$ dans les germoirs traditionnels). Une même densité de semis dans les deux types de germoirs pourrait ressortir l'efficacité des germoirs modernes.

Les résultats obtenus du test de croissance des plantules de A. апnиa L. ont montré qu'il existe un effet significatif du substrat utilisé. La croissance des plantules sur sol ferrallitique est meilleure à celle obtenue $\mathrm{au}$ niveau du sol sableux. Les sols ferrallitiques ont en effet, une meilleure capacité de rétention d'eau et des propriétés physico-chimiques, notamment les cations échangeables, la matière organique et l'azote total (tableau 1) qui favoriseraient la meilleure croissance et le bon développement des plantules de A. аппиа L. anamed. Des résultats similaires sont obtenus par Müller et Brandes (1997) sur la variété sauvage de $A$. апnиa. Ces auteurs ont noté une hauteur 
Tableau 6: Moyenne $\left(\mathrm{m}, \mathrm{g} / \mathrm{m}^{2}\right)$ et coefficient de variation $(\mathrm{cv}, \%)$ des variables de production par plant selon l'écartement sur sol ferrallitique.

\begin{tabular}{|c|c|c|c|c|c|c|}
\hline \multirow{3}{*}{ Variable $\left(\mathrm{g} / \mathrm{m}^{2}\right)$} & \multicolumn{6}{|c|}{ Ecartement } \\
\hline & \multicolumn{2}{|c|}{$0,8 \mathrm{~m} \times 0,6 \mathrm{~m}$} & \multicolumn{2}{|c|}{$0,8 \mathrm{~m} \times 0,8 \mathrm{~m}$} & \multicolumn{2}{|c|}{$1 \mathrm{~m} \times 1 \mathrm{~m}$} \\
\hline & $m$ & $c v$ & $m$ & $c v$ & $m$ & $c v$ \\
\hline PFFeuil & $443.2 \mathrm{a}$ & 66.5 & $185.6 \mathrm{c}$ & 59.7 & $236.8 b$ & 87.3 \\
\hline PFTige & $66.6 \mathrm{a}$ & 57.9 & $36.3 b$ & 58.9 & $38.8 \mathrm{~b}$ & 74.3 \\
\hline PFracine & $74.1 \mathrm{a}$ & 80.1 & $33.1 \mathrm{~b}$ & 60.4 & $46.8 \mathrm{~b}$ & 91.3 \\
\hline PSFeuil & $219.9 a$ & 65.4 & $88.1 \mathrm{~b}$ & 53.6 & $99.6 \mathrm{~b}$ & 88.9 \\
\hline PStige & $44.1 \mathrm{a}$ & 59.6 & $21.9 \mathrm{~b}$ & 61.6 & $20.4 b$ & 72.8 \\
\hline PSracine & $48.3 \mathrm{a}$ & 78.3 & $19.4 b$ & 56.0 & $24.4 \mathrm{~b}$ & 87.8 \\
\hline BiomtF & $583.9 \mathrm{a}$ & 62.6 & $255.0 \mathrm{~b}$ & 54.5 & $322.4 b$ & 83.8 \\
\hline BiomtTs & $312.4 \mathrm{a}$ & 62.3 & $129.4 \mathrm{~b}$ & 51.4 & $144.0 \mathrm{~b}$ & 83.0 \\
\hline
\end{tabular}

Pour chaque paramètre, les chiffres de même lettre ne sont pas significativement différents. PFFeuil: poids frais des feuilles; PFtige: poids frais de tige; PFrac: poids frais de racine; BiomtF: biomasse totale fraîche; PSFeuil: poids sec des feuilles; PStige: poids sec de tige; PSrac: poids sec de racine ; BiomTs: biomasse totale sèche.

moyenne des plants de $0,23( \pm 0,34) \mathrm{m}$ sur sol sableux contre $0,81( \pm 0,18)$ m sur terreau. De plus, les résultats de ces auteurs indiquent que la croissance des jeunes plants avait une allure logistique sur les deux types de substrat avec toutefois une vitesse $r$ de croissance logistique plus élevée sur terreau ( $r=0,124$ contre 0,109 sur sol sableux)

Par ailleurs, les plants de A. апnиa obtenus de cette étude ont présenté à la plantation sur terre ferme une hauteur maximale allant de $1,46 \mathrm{~m}$ à $1,60 \mathrm{~m}$; ce qui est différent de ceux obtenus par Hans (2005) et Barrié (2006) avec une hauteur maximale de $2,50 \mathrm{~m}$ et $3 \mathrm{~m}$, respectivement lors des expériences sur la plante $A$. апnиa L. anamed. La différence notée s'explique par l'apport d'engrais dans leur expérimentation. En effet Müller et Brandes (1997) ont montré qu'un apport croissant d'engrais aux jeunes plants de A. аппиа (30, 60 et $120 \mathrm{~kg} \mathrm{~N} / \mathrm{ha})$ induisait aussi un accroissement en hauteur, en biomasse sèche et en nombre de bourgeons mais jusqu'à une limite déterminée par la fonction de Mitscherlich (Richter, 1985).

Les résultats de la présente étude ont montré que la biomasse totale ainsi que le poids moyen des feuilles, tiges et racines obtenus à la plantation sur terre ferme était plus élevés pour l'écartement $0,8 \mathrm{~m} \mathrm{x} 0,6 \mathrm{~m}$ (8,33 plants $/ \mathrm{m}^{2}$, tableau 6). Les autres densités de plantation (4 et 6,25 plants $/ \mathrm{m}^{2}$ ) présentent pratiquement les mêmes valeurs de biomasse. Ces résultats permettent de déduire que l'intensité de la compétition intra-spécifique d'un écartement à un autre, du moins pour les écartements considérés dans la présente étude ne semble pas affecter la production de biomasse des plants de A. апnиа.

\section{Conclusion}

Les résultats obtenus de cette étude permettent alors de noter qu'une bonne production de biomasse de $A$. апnиа en Afrique de l'Ouest et particulièrement au Bénin pourrait être obtenue en installant des pépinières dans des germoirs traditionnels fait de matériaux locaux avec un substrat ferrallitiques. Les jeunes plants issus des pépinières pourront être installés sur sol ferralitique avec une densité de plantation de 8,3 plants $/ \mathrm{m}^{2}$. Ces conclusions sont toutefois valables dans les limites de l'étude et des expérimentations ultérieures en considérant que d'autres types de sols et d'écartements permettront de tirer des conclusions plus générales.

\section{REFERENCES}

Barrié P. 2006. L'artemisia апnиа ou comment lutter contre le paludisme. 
Source : http:// www acp-paludisme.com. Consulté le 06 janvier 2007.

Dreuilhe P. 2005. La vaccination contre le paludisme. Source: http:// www acppaludisme.com. Consulté le 14 novembre 2006.

Ferreira JFS, Janick J. 1995. Floral morphology of Artemisia annua with special reference to trichomes. Int. J. Plant Sci., 156: 807-815.

Ferreira JFS, Simon JE, Janick J. 1997. Artemisia annua: botany, horticulture, pharmacology (a review). Horticultural Reviews., 19: 319-371.

Guynnot R. 2005. Paludisme: Novartis débordé. Source: http:// www. jeuneAfrique.com. Consulté le 14 novembre 2006.

Hans M. 2001. La Médecine Naturelle Tropicale Traitements. Publication Anamed: Allemagne; 43p.

Hans M. 2003. Médecine Naturelle Tropicale. Publication Anamed: Allemagne.

Hans M. 2005. Artemisia annua anamed: Malaria et la Médecine Naturelle. Publication Anamed: Nr 203 (7-aa-fra), Allemagne.

Hans M, Keith L. 2006. Thé à Artemisia annua. Source: http://www.anamed.net. Consulté le $1^{\text {er }}$ février 2007.
Klayman DL. 1993. Artemisia annua from weed to respectable antimalarian plant. In Human Medicinal Agents from Plants, Kinghorn AD, Balandrin MF (eds). ACS Symposium Series 534: Washington DC; $243-255$.

Lacroix C. 2004. Le Petit Larousse Illustré 2005. Dictionnaire. $\left(100^{\text {th }}\right.$ edn $)$. Larousse.

Meusel H, Jäger E. 1992. Vergleichende Chorologie der Zentraleuropäischen Flora. Bd. III. Text: Stuttgart, New York.

Müller M, Brandes D. 1997. Growth and development of Artemisia annua $l$. on different soil types. Verhandlungen der Gesellschaft für Ökologie, 27: 453-460.

Richter O. 1985. Simulation des verhaltens ökologischer systeme.-VCH. Weinheim.

Organisation Mondiale de la Santé, 2006. Guidelines for the Treatment of Malaria. OMS: Genève.

SAS Inst. 1999. SAS/STAT User's Guide. SAS Institute. Cary NC.

Sogansa N. 2003. Influence de deux types de sols (ferrallitique et minéral brut) sur le bilan hydrique et la biodiversité des champignons mycorhiziens sous plantation d'Acacia auriculoformis et d'Acacia mangium. Thèse d'Ingénieur Agronome. UAC/FSA, Abomey-Calavi. 